\title{
TinyOS-Based Gateway for Underwater Acoustics/Radio Frequency Communication
}

\author{
Phil-Jung Yun, Changhwa Kim, Sangkyung Kim, \\ Seung-Jae Lee, and Yong-Man Cho \\ Department of Computer Science \& Engineering, \\ Kangnung National University \\ Gangneung Daehangno 120, Gangneung-Si, Gangwon-Do, 210-702, Korea \\ Tel.: +82-33-640-2897; Fax: +82-33-640-2899 \\ mars@cs. kangnung.ac.kr, \{kch, skkim98, silveree, ymcho\}@kangnung.ac.kr
}

\begin{abstract}
Currently, UWASN (Underwater Acoustic Sensor Network) has been researched as a branch of sensor networks. UWASNs use a transmission media different from terrestrial wireless sensor networks. That is, the former uses acoustic waves and the latter uses radio waves for communication. G/W (Gateway)s in UWASNs are nodes for relaying data transmission between a UWASN and a terrestrial wireless sensor network. So, it is necessary to design and realize the UWA communication module with the UWA/RF (Underwater Acoustics/Radio Frequency) protocol stack for the underwater environment. As TinyOS used most widely at operating system for sensor networks is not considering the underwater acoustic communication, the functions supported by it cannot be used. Therefore, The TinyOS-based UWA/RF G/W system and prototype suitable for the characteristic of UWASN is designed and realized in this paper.
\end{abstract}

Keywords: Underwater Acoustic Sensor Network, Underwater Acoustics/Radio Frequency, Gateway, TinyOS.

\section{Introduction}

As acoustic waves are attenuated faster than radio waves in the air and vice versa in the underwater, it is needed to use more proper method for communication in each environment. Currently, for radio wave communication in the underwater, very low frequency $(30-300 \mathrm{~Hz})$, very long antennae and very high transmission power are needed. Although optical waves are used, it suddenly diminishes in the underwater. Therefore, acoustic waves are used for underwater communication [1].

Acoustic waves have very different characteristics from radio waves in speed, bandwidth and transmission energy [2], [3], [4], [5]. It has slower speed, narrower bandwidth and fewer channels than radio waves [6], [7], [8]. It also needs more transmission power than radio wave [9].

Underwater acoustic communication has many different characteristics and these are listed below 
- Communication Range: As low frequency waves are used in UWASN, it has longer communication range than radio waves.

- Communication Power: Acoustic communication needs more transmission energy.

- Channel: There are only a few channels available in acoustic communication. Communication speed is very slow, variable and multipath and fading problems might happen.

- Bandwidth: It has very low bandwidth.

- Cost: The costs of sensor nodes and acoustic modems are high.

- Deployment: The density of sensor nodes affects the cost and the communication range. Multi-hop communication is more effective than direct communication in transmission energy if the distance between two nodes is long [10]. A sparse UWASN increases the communication cost and a dense UWASN increases the communication cost. Therefore, density of a UWASN should be determined by application objectiveness of the UWASN.

- $\quad$ Energy Limit: Energy of UWASNs is very limited because it is very hard to recharge the batteries.

- High Error Rate: The bit error rate in acoustic communication is high.

UWASNs have been studied with the focusing on network and MAC layer for considering the characteristics of UWASNs and acoustic modems for UWA communication. Ethem M.Sozer and Milica Stojanovic has developed Reconfigurable Acoustic Modem (rModem) [11], [12] and Jack Wills, Wei Ye and John Heidemann have developed Low-Power Acoustic Modem for Dense deployment for UWASN [13].

However, although it is necessary to send queries from terrestrial centers to UWASN sensor nodes and to send data from UWASN sensor nodes to terrestrial centers, the researches for relaying data transmission between UWASNs and the ground network are very rare. We have tried to study and implement UWA/RF G/W system before developing UWA/CDMA or UWA/satellite G/W system.

The objective of the UWA/RF G/W system is relaying data transmission between a UWASN and a terrestrial center. Sensed data in sensor nodes are transferred to a UWA/RF G/W via UWA communication and the data received by the acoustic modems of sensor nodes are transferred from the UWA/RF G/W to a terrestrial center via RF communication. The UWA/RF G/W system is positioned at the relay point and it must be designed and realized with consideration for both air and underwater environment because it uses both radio waves and acoustic waves. Additionally, the acoustic modem for physical communication and the protocol stack for effective communication is also needed.

Selection of the operating system is very important in developing the UWA/RF G/W system. It affects design, realization method, effectiveness and stability of the system. Therefore, the UWA/RF G/W is developed in this paper based on TinyOS 2.0.0 Beta which has much strength for sensor networks.

The rest of this paper is constructed as follows. In section 2, requirements for UWA/RF G/W are presented and section 3 illustrates UWA/RF sensor network 
system environment. Section 4 shows the UWA/RF G/W system design and section 5 describes the UWA/RF G/W system prototype. Finally, section 6 concludes and finalizes this paper.

\section{Requirements for UWA/RF G/W System}

The requirements for design and realization of the UWA/RF G/W system are divided into four categories as follows:

First, the acoustic modem and UWA communication module considering the underwater must be realized because underwater sensor nodes communicate each other using acoustic waves.

Second, the UWA/RF G/W protocol stack considering underwater environment and UWASN characteristics should be designed. Because the sensor network standard specifications currently used, i.e. IEEE 802.15.4 and Zigbee, are not designed for underwater environment and UWASN characteristics.

Third, the management functions for communication modules must be analyzed and realized because UWA/RF G/W nodes communicate using both radio waves and acoustic waves.

Finally, a proper operation system be selected considering stability, reconfiguration cost, etc,. TinyOS used in this paper is a component-based operation system for sensor networks and supports reusability, compatibility, portability and productivity.

\section{UWA/RF Sensor Network Architecture}

Fig. 1 shows the UWA/RF sensor network architecture. It transfers sensed data to terrestrial centers and transfers queries to sensor nodes. And it consists of UWA sensor nodes, UWA/RF G/W nodes and RF relay nodes.

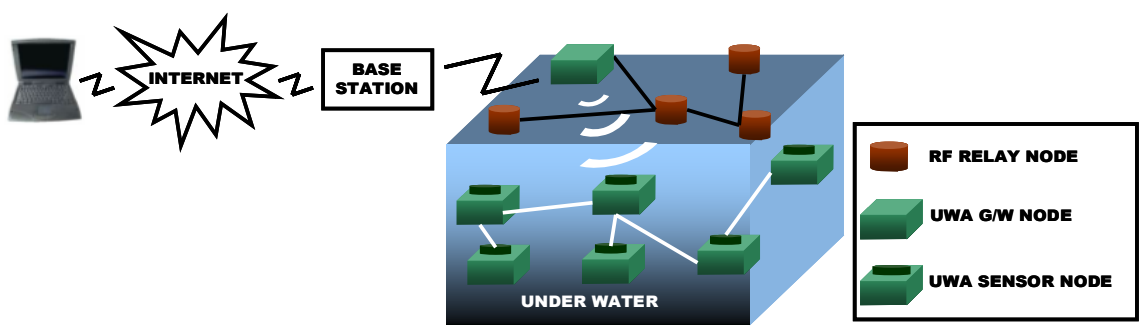

Fig. 1. UWA/RF Sensor Network Architecture

UWA sensor nodes sense underwater environment and communicate with other UWA sensor nodes. UWA/RF G/W nodes convert radio waves to acoustic waves and vice versa. And RF relay nodes relay UWA/RF G/W nodes and terrestrial centers when a UWA/RF G/W cannot communicate directly with a terrestrial center. 


\section{Design of TinyOS-Based UWA/RF G/W Design}

The protocol stack of the UWA/RF G/W and the UWA/RF G/W system are designed in this section. Fig. 2 shows the protocol stack of the UWA/RF G/W and it consists of Gateway Module, Communication Module Transmission Manager, UWA MAC layer, UWA PHY layer and Communication Module Reception Manager.

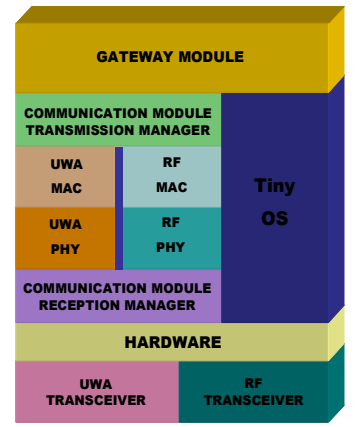

Fig. 2. UWA/RF G/W Protocol Stack

Gateway Module supports the functions that control and manage the UWA/RF G/W node. Communication Module Transmission Manager solves the problems that might occur during multiple instances of Communication Module being executed. The UWA MAC layer provides MAC functions which are proper to underwater environment. The UWA PHY layer controls and manages hardware, ensures link quality and selects channels. Communication Module Reception Manager prevents problems which might occur during reception with multiple instances of Communication Module.

\subsection{Gateway Module}

Gateway Module is designed like Fig. 3 and the ITRC_GATEWAYMODULE component provides functions that enable to control the UWA/RF G/W node according to the event that occurs in the UWA/RF G/W node. The events are reception completion events of messages for RF or for UWA.

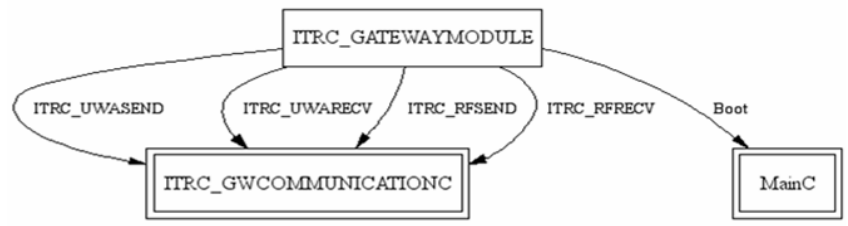

Fig. 3. Gateway Module Design

The event caused by reception completion event for RF message occurs when a query sent to sensor nodes arrives and the reception completion event for UWASN message occurs when a response of a query or a sensed data sent to a terrestrial center arrives. 


\subsection{Communication Module Transmission Manager}

Fig. 4 shows Communication Module Transmission Manage and consists of ITRC_GWCOMMUNICATIONP and ITRC_TRANSMISSIONMANAGER components.

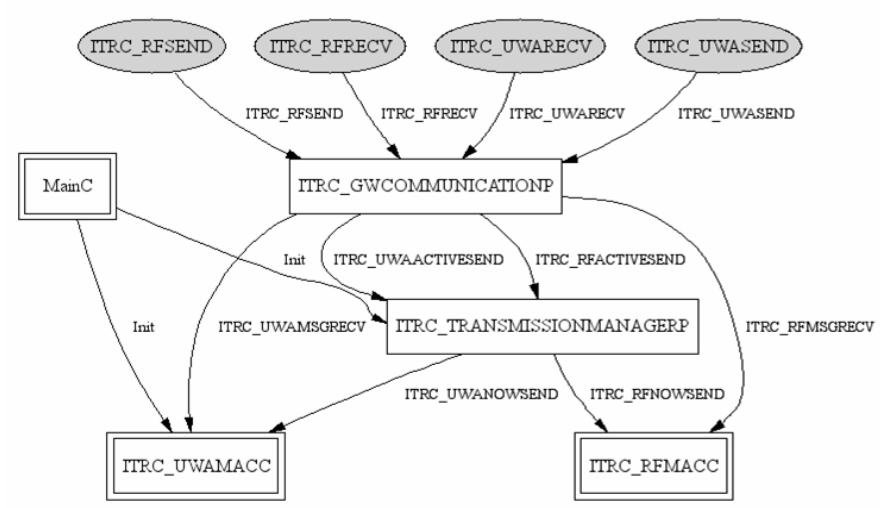

Fig. 4. Design of Communication Module Transmission Manager

ITRC_GWCOMMUNICATIONP transfers a request or an event to each component after it determines whether it is a transmission request to RF, UWA communication module or an event for reception completion.

ITRC_GWCOMMUNICATIONMANAGER serializes the transmission requests from RF and UWA and it enables to be processed in turn. The scheduler of TinyOS also supports multiple transmission requests [14]. If current transmission message is a request to the RF communication module, the request is delivered to ITRC_RFMACC. Or if it is a request to the UWA communication module, it is delivered to ITRC_UWAMACC.

Table 1. Interface supported by Communication Module Transmission Manager

\begin{tabular}{|c|c|}
\hline Interface & Functions \\
\hline ITRC_UWASEND & $\begin{array}{l}\text { Processing transmission message for reporting } \\
\text { transmission completion for UWA messages }\end{array}$ \\
\hline ITRC_RFSEND & $\begin{array}{l}\text { Processing transmission message for reporting } \\
\text { transmission completion RF messages }\end{array}$ \\
\hline ITRC_UWARECV & $\begin{array}{l}\text { Reporting reception completion of UMA messages } \\
\text { and delivering received messages }\end{array}$ \\
\hline ITRC_RFRECV & $\begin{array}{l}\text { Reporting reception completion of RF messages and } \\
\text { delivering received messages }\end{array}$ \\
\hline ITRC_UWAACTIV & $\begin{array}{l}\text { Scheduling UWA transmission requests with } \\
\text { serialization }\end{array}$ \\
\hline ITRC_RFACTI & Scheduling RF transmission requests with serialization \\
\hline
\end{tabular}


The interfaces that Communication Module Transmission Manager supports are listed in Table 1.

\subsection{UWA MAC Layer}

Fig. 5 shows the design of the UWA MAC layer. It consists of ITRC_UWAMACP and ITRC_UWACHANNELACCESSP components.

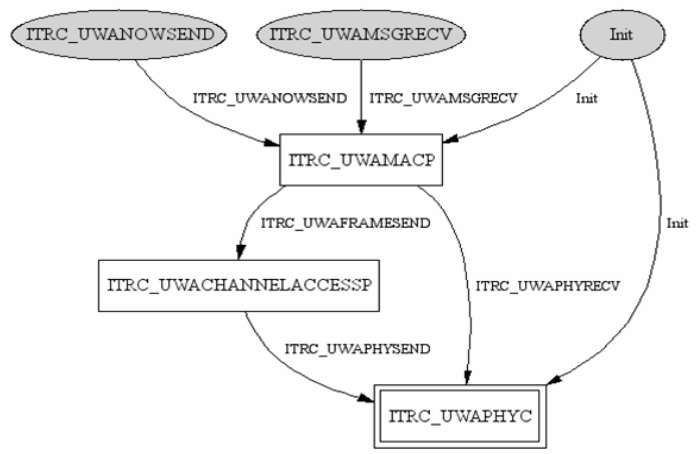

Fig. 5. Design of UWA MAC Layer

ITRC_UWAMACP frames messages received with transmission request from Communication Module Transmission Manager. The frames include a sequence number, CRC and frame size. On receipt of a frame, it delivers received frames to the next higher layer after error check.

ITRC_UWACHANNELACCESSP determines the channel access method for transmitting a frame generated at ITRC_UWAMACP and transmits the frame by the method.

Table 2. Interfaces provided by the UWA MAC layer

\begin{tabular}{ll}
\hline \multicolumn{1}{c}{ Interface } & \multicolumn{1}{c}{ Functions } \\
\hline ITRC_UWANOWS & Converting messages to frames \\
END & $\begin{array}{l}\text { Processing the transmission request for UWA frame } \\
\text { with defined channel access method by the system. } \\
\text { ITRC_UWAFRAM } \\
\text { ESEND }\end{array}$ \\
$\begin{array}{l}\text { Reporting transmission completion } \\
\text { Reporting reception of UWA frame. Deliverring the } \\
\text { payload after checking errors of a received UWA frame }\end{array}$ \\
\hline
\end{tabular}

Table 2 shows the list interfaces which the UWA MAC layer provides in order to process transmission request from Communication Module Transmission Manager. 


\subsection{UWA PHY Layer and Communication Module Reception Manager}

The UWA PHY layer and Communication Module Reception Manager are designed like Fig. 6. The UWA PHY layer consists of the ITRC_UWAPHYP component and Communication Module Reception Manager consists of the ITRC_UWARECEPTIONMANAGERP component.

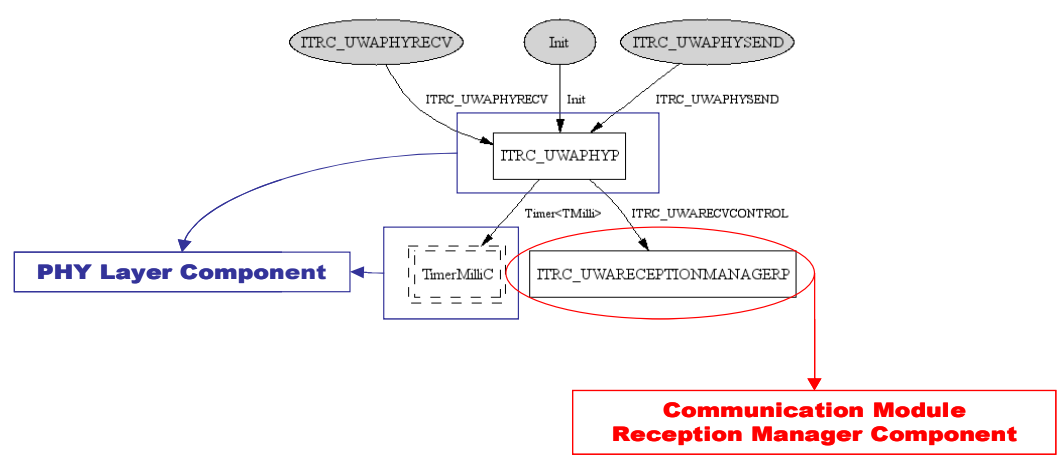

Fig. 6. Design of UWA PHY Layer and Communication Module Reception Manager

ITRC_UWAPHYP transfers frames through the acoustic modem after transforming it to PDU-1(Protocol Data Unit-1) given a transmission request from the UWA MAC layer. If there is a received PDU-1, it delivers a received PDU-1 with the report that it receives PDU-1 from the UWA MAC layer.

On receiving a frame from the acoustic modem, ITRC_UWARECEPTION MANAGERP resolves problems that might occur by multiple instances of Communication Module Reception Manager by disabling the reception interrupt from all of instances of Communication Module Reception Manager except the acoustic modem during receiving a frame.

Table 3. Interfaces supported by UWA PHY Layer and Communication Module Reception Manager

\begin{tabular}{ll}
\hline \multicolumn{1}{c}{ Interface } & \multicolumn{1}{c}{ Functions } \\
\hline ITRC_UWAPHYSE & $\begin{array}{l}\text { Transmitting transmission requests for a UWA frame } \\
\text { with the acoustic modem. Reporting transmission } \\
\text { completion } \\
\text { Reporting reception of PDU-1. Delivering the received } \\
\text { ITRC_UWAPHYRE }\end{array}$ \\
$\begin{array}{l}\text { PDU-1 } \\
\text { ITRC_UWARECV }\end{array}$ & $\begin{array}{l}\text { Disabling interrupts from other communication } \\
\text { modules after detecting reception at the UWA PHY } \\
\text { CONTROL }\end{array}$ \\
\hline
\end{tabular}

The interfaces supported by the UWA PHY layer and Communication Module Reception Manager are listed in Table 3. The interfaces supported by the UWA PHY layer processes transmission requests from the UWA MAC layer. 


\section{Implementation of TinyOS-Based UWA/RF G/W Prototype}

UWA/RF G/W prototype based on TinyOS using a UWASN acoustic modem which can be used at UWASN nodes is realized in this section.

\subsection{Overview of the Acoustic Modem}

The characteristics of the acoustic modem used in this research are as follows.

- $\quad$ Operating Voltage: $3.3 \mathrm{~V}$

- Frequency: $40 \mathrm{KHz}(u l t r a$ sonic wave)

- Modulation Method: Amplitude Shift Keying

- Communication Mode: Uni-directional

- Data rate: $100 \mathrm{bps}$.

\subsection{The Algorithm for Gateway Module}

The pseudo-code for Gateway Module is shown in Fig. 7. The ITRC_RFRECV event means completion of receiving a RF message. If this event occurs, the received RF message is delivered to Gateway Module as a parameter. In the case that a received RF message means a query, this message is delivered to UWASN nodes through UWA Communication Module in UWA/RF G/W. Transmission request is delivered to Communication Module Transmission Manager using ITRC_UWASEND.

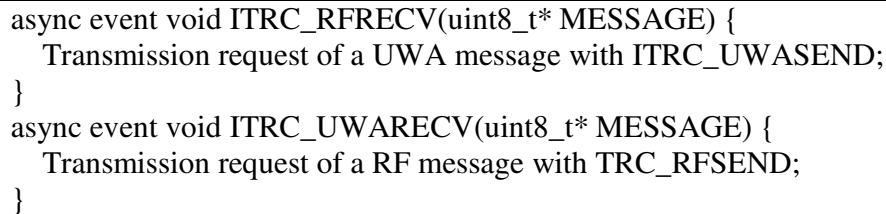

Fig. 7. The Pseudo-code for Gateway Module

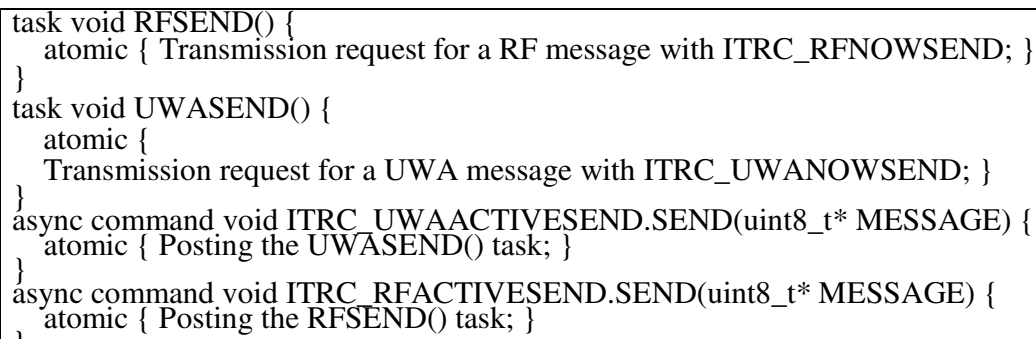

Fig. 8. The Pseudo-code for Gateway Module

The ITRC_UWARECV event means completion of receiving a UWA message. If this event occurs, the received UWA message is delivered to Gateway Module as a 
parameter. In the case that a received RF message means a response or sensed data from a UWA sensor node, this message is delivered to a terrestrial center through RF Communication Module in UWA/RF G/W. Transmission request is delivered to Communication Module Transmission Manager using ITRC_RFSEND.

The pseudo-code for Communication Module Transmission Manager is presented in Fig. 8. ITRC_UWAACTIVESEND.SEND () is called by transmission request for a UWA message at Gateway Module and ITRC_RFACTIVESEND. SEND() is called by transmission request for a RF message at Gateway Module. Because all the requests are serialized by the scheduler in TinyOS, a task with processing parts of a function is posted on the scheduler when a transmission request occurs.

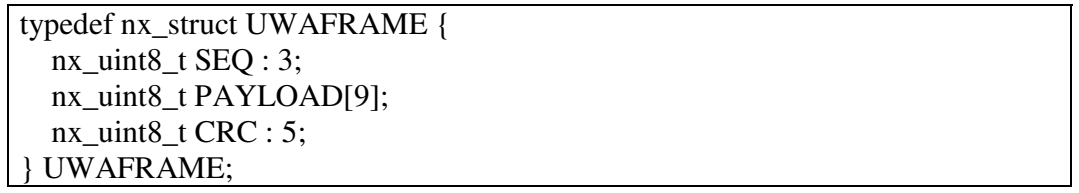

Fig. 9. UWA Frame Structure

Fig. 9 shows the frame structure of UWA frames. This frame consists of 3 bits sequence number, 5 bits CRC and 9 bytes payload.

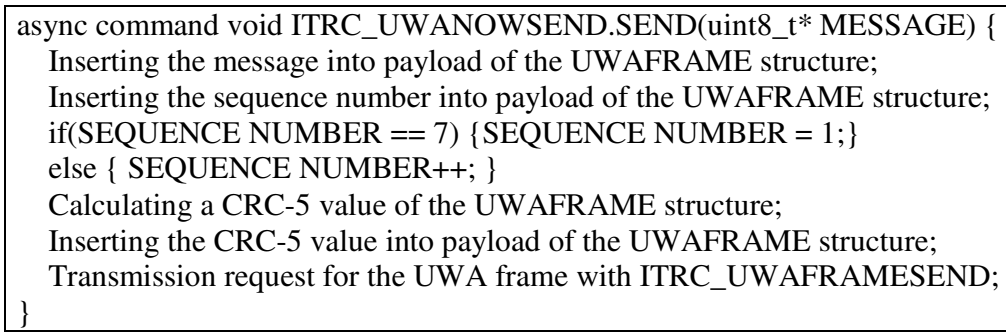

Fig. 10. The Pseudo-code for transmission in the UWA MAC layer

The pseudo-code for transmission in the UWA MAC layer is like Fig. 10. When ITRC_UWAPHYSEND.SEND() is called by transmission request from Communication Module Transmission Manager, this function receives a UWA frame in the parameter. ITRC_UWANOWSEND.SEND () frames the received UWA message. Then, it inserts the message into payload of the frame and assigns the sequence number with SEQ. Calculated CRC value are also inserted into the frame. Finally, generated frame and transmission request are delivered to the UWA PHY layer.

The pseudo-code for the UWA PHY layer is described in Fig. 11. When ITRC_UWAPHYSEND.SEND () is called by transmission request from the UWA MAC layer, this function receives a UWA frame in the parameter. If the state of the acoustic modem is not IDLE, it discards the request because the acoustic modem is currently receiving some data. If not, it starts to transmit the frame. In this case, the state of the acoustic modem is set to BUSY, the timer is reset according to the 
communication cycle of the acoustic modem and one by one bit at a time is transmitted as the timer is fired. After transmitting all the frame, the timer is stopped and the state of the acoustic modem is set to IDLE. Finally, it causes the

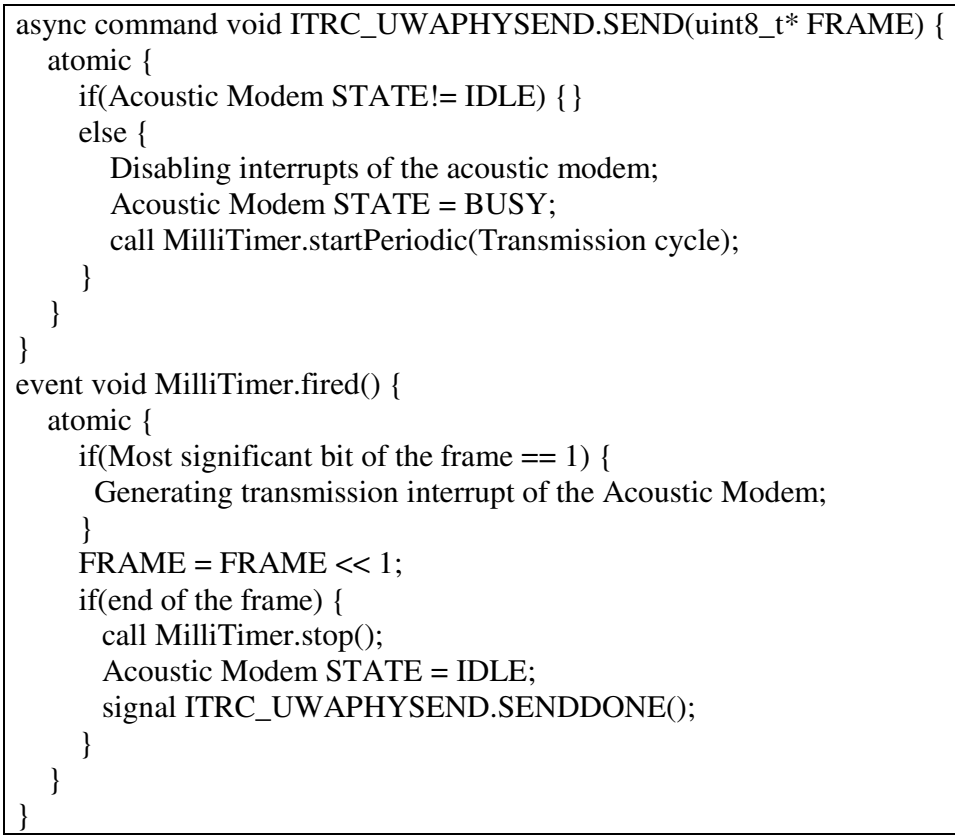

Fig. 11. UWA PHY Send Algorithm

UWAPHYSEND.SENDDONE event so that reports transmission completion to the UWA MAC layer.

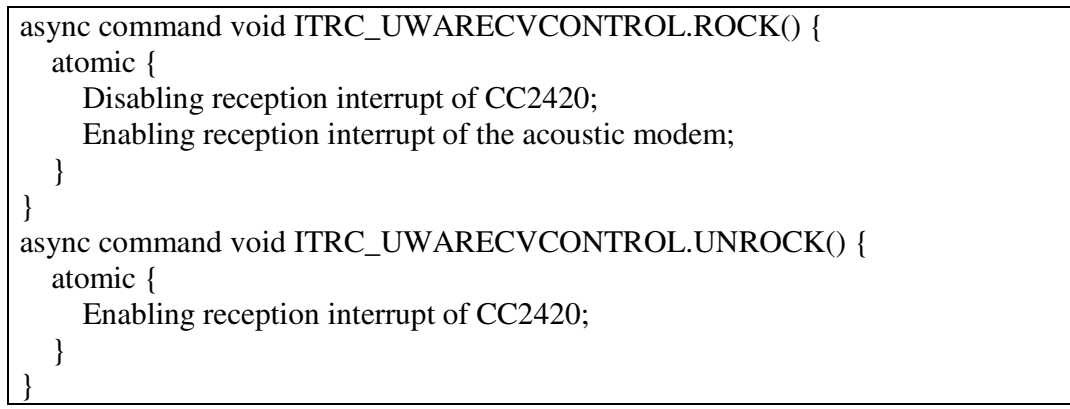

Fig. 12. Communication Module Reception Manager Algorithm

The pseudo-code for Communication Module Reception Manager is described in Fig. 12. On receiving the first bit of a frame at the acoustic modem, ITRC_UWARECVCONTROL.ROCK() is called. Reception completion report for 
the first bit of a frame from the acoustic modem is a signal that it receives a bit of a frame and means that it starts receiving a frame. In this case, it disables reception interrupt from all other instances of Communication Module. On finishing reception task for a frame, ITRC_UWARECVCONTROL. UNROCK() is started. This function solves problems that might occur with multiple instances of Communication Module by enabling reception interrupt.

\section{Conclusions and Future Works}

In this paper, relaying method between UWASNs and terrestrial centers using $\mathrm{UWA} / \mathrm{RF} \mathrm{G} / \mathrm{W}$ is proposed. UWA/RF G/W based on TinyOS is designed with consideration for the underwater and the characteristics of UWASN. The UWA/RF G/W protocol stack for effective communication in the UWA/RF G/W system is also designed and realized. In addition, Communication Module Transmission Manager and Communication Module Reception Manager are designed and realized to support managing multiple instances of Communication Module.

Effective Channel Access Methods and the network layer for the UWA/RF G/W system and design and realization of components for UWASN systems will be explored in the future.

Acknowledgments. This research was supported by the MIC (Ministry of Information and Communication), Korea, under the 2006 ITRC (Information Technology Research Center) contract number IITA-2006-C1090-0603-0044 support program supervised by the IITA(Institute of Information Technology Assessment).

\section{References}

1. Stojanovic, M.: Acoustic (underwater) Communications. In: Proakis, J.G. (ed.) Encyclopedia of Telecommunications, Wiley, New York (2003)

2. Akyildiz, I.F., Su, W., Sankarasubramaniam, Y., Cayirci, E.: A Survey on Sensor Networks. IEEE Communications Magazine (2002)

3. Proakis, J.G., Sozer, E.M., Rice, J.A., Stojanovic, M.: Shallow Water Acoustic Networks. IEEE Communications Magazine (2001)

4. Jurdak, R., Lopes, C.V., Baldi, P.: Battery Lifetime Estimation and Optimization for Underwater Sensor Networks. IEEE Press, New York (2004)

5. Sozer, E., Stojanovic, M., Proakis, J.: Underwater Acoustic Networks. IEEE Journal of Oceanic Engineering, 70-83 (2000)

6. Freitag, L., Stojanovic, M.: Acoustic Communications for Regional Undersea Observatories. In: Proceedings of Oceanology International, London, UK (2002)

7. Freitag, L., Stojanovic, M., Singh, S., Johnson, M.: Analysis of Channel Effects on Directsequence and Frequency-hopped Spread-spectrum Acoustic Communication. IEEE Journal of Oceanic Engineering , 586-593 (2001)

8. Stojanovic, M.: Recent Advances in High-Speed Underwater Acoustic Communications. IEEE Journal of Oceanic Engineering , 125-136 (1996) 
9. Akyildiz, I.F., Pompili, D., Melodia, T.: State-of-the-Art in Protocol Research for Underwater Acoustic Sensor Networks. In: International Workshop on Underwater Network, MobiCom, pp. 7-16 (2006)

10. Pottie, G.J., Kaiser, W.J.: Embedding The Internet: Wireless Integrated Network Sensors. Communications of the ACM, 51-58 (2000)

11. Sozer, E.M., Stojanovic, M.: Time Synchronization for High Latency Acoustic Networks. In: Proceedings of the International Symposium on Unmanned Untethered Submersible Technology(UUST), Lee, New Hampshire, USA (2005)

12. Sozer, E.M., Stojanovic, M.: Reconfigurable Acoustic Modem for Underwater Sensor Networks. In: International Workshop on Underwater Network, MobiCom, pp. 101-104 (2006)

13. Wills, J., Ye, W., Heidemann, J.: Low-Power Acoustic Modem for Dense Underwater Sensor Networks. In: International Workshop on Underwater Network, MobiCom, pp. 7985 (2006)

14. Levis, P., Sharp, C.: Schedulers and Tasks, http://tinyos.cvs.sourceforge.net/*checkout*/tinyos/tinyos-2.x/doc/html/tep106.html 